\title{
Distance between Separating Circles and Points
}

\author{
Peter Veelaert \\ University College Ghent, Engineering Sciences - Ghent University Association, \\ Schoonmeersstraat 52, B9000 Ghent, Belgium \\ Peter.Veelaertahogent.be
}

\begin{abstract}
The family of separating circles of two finite sets $S_{1}$ and $S_{2}$ in the plane consists of all the circles that enclose $S_{1}$ but exclude $S_{2}$. We prove that the maximum and minimum distance between a point $p$ and any separating circle in this family can be found by examining only a finite subset of circles, although the family itself is infinite. In addition, we introduce the concept of elementary circular separations to clarify some of the properties of separating circles.
\end{abstract}

\section{Introduction}

We consider the problem of finding the shortest and largest distance between a point and a family of separating circles. More precisely, let $S^{-}$and $S^{+}$be two sets of planar points. We consider the family of separating circles for which the points of $S^{-}$always lie inside the circles and the points of $S^{+}$outside. We want to show that shortest and largest distance between a planar point and any member of this family can be found in a finite number of steps, although the family itself is infinitely large. This result is also of use for finding distances between two families of separating circles, or between a straight line and a family of circles.

O'Rourke et al already noted that there is a unique smallest separating circle, which can be found by convex programming [7]. The largest separating circle cannot be found by convex programming and is not always unique. They also showed that any largest separating circle of finite radius must either pass through 3 points of $S^{+}$, or must pass through 2 points of $S^{+}$and one of $S^{-}$. Thus, the largest circle can be found by examining all the 3-point subsets of $S^{-} \cup S^{+}$.

Our results on separating circles are also relevant for digitized circles. In the past two distinct views have been developed on digital curves [6]. In one view one regards a digital curve as a set of points for which there is a continuous curve that is passing close to these points. This is the preferable viewpoint when regarding a digital curve as the digitization of a continuous curve. A second view is preferred, when one considers a digital curve as the boundary of a digital set. Then the digital set can be separated from its complement by a continuous curve. The separation/closeness dichotomy reappears when we examine the basic properties of digital curves. Elemental subsets are the smallest subsets of a curve for which it is meaningful to define closeness. Thus, an elemental circular subset has 4 points, since a circle can always pass at zero distance from 3 points. The word elemental comes from robust estimation theory where it refers to subsets that are just large enough to produce an estimate. As for separation, we will introduce the concept of elementary circular separations, containing as few as 3 points.

I. Debled-Rennesson et al. (Eds.): DGCI 2011, LNCS 6607, pp. 346-357, 2011.

(C) Springer-Verlag Berlin Heidelberg 2011 
Several authors have proposed algorithms to determine circular separability [3-8]. Invariably, either the parameter domain of the separating circles, or its projection on the plane of circle centers, plays an important role in these algorithms. In this work we shall prove some additional results that link the parameter domains to elementary circular separations.

\section{Elementary Circular Separations and Parameter Domains}

Elementary circular separations are introduced to provide a means of separating the points of a set $S=S^{-} \cup S^{+}$unambiguously by circles, even when a circle passes through some points of $S$. Since 3 points uniquely define a circle, the definition of elementary circular separations is based on 3-point subsets, although more than 3 points are allowed when they are circular by coincidence.

Let $R \subseteq S$ be a set of 3 or more points lying on a common circle conveniently denoted by $C_{R}$. Let $\sigma_{R}: R \rightarrow\{+,-\}$ be a function that attributes a sign to each point of $R$.

Definition 1. The sign function $\sigma_{R}$ is called an elementary circular separation if there exists a circle $C$ not equal to $C_{R}$, such that all the points of the preimage $\sigma_{R}^{-1}(+)$ lie outside $C$ and all the points of the preimage $\sigma_{R}^{-1}(-)$ lie inside $C$.

By definition $C$ does not pass through any of the points of $R$. We will often use the shorthands $R^{+}=\sigma_{R}^{-1}(+)$, and $R^{-}=\sigma_{R}^{-1}(-)$. Note that although the total number of points must be at least 3 , one of the subsets $R^{+}$or $R^{-}$may be empty.

Since the circle $C_{R}$ only passes through the points in $R$ and not through any other point of $S$, the separation function $\sigma_{R}$ can be extended to a function $\sigma_{S}$ over the entire set in an unambigous way, where $\sigma_{S}$ attributes the minus sign to a point that lies in the interior of $C_{R}$, and the plus sign when it is exterior. We will use the shorthands $S^{+}=\sigma_{S}^{-1}(+)$, and $S^{-}=\sigma_{S}^{-1}(-)$.

\subsection{Path-Connected Parameter Sets}

The extension of $\sigma_{R}$ from an elementary separation to the entire set is straightforward. We will show that the converse is also true. When a family is defined as the set of circles that separate $S$ into given parts $S^{+}$and $S^{-}$, we show that this family can always be represented by an elementary circular separation. To accomplish this we define pathconnected sets in the parameter space of separating circles. Let $S$ be a non-empty finite set of points in the plane. A circle is defined by an equation of the form

$$
(x-a)^{2}+(y-b)^{2}-r^{2}=0,
$$

which can always be rewritten as

$$
x^{2}+y^{2}-2 a x-2 b y+c=0,
$$

with $a^{2}+b^{2}-r^{2}=c$. Conversely, (1) corresponds to a real circle provided $c \leq a^{2}+b^{2}$. We are interested in circles that separate the points of $S$ unambiguously, which is the case if they do not pass through any of the points of $S$. This leads to a 3D parameter space from which some planes are removed. 
Definition 2. Let $S$ be a finite subset of $\mathbb{R}^{2}$ and let $U \subset \mathbb{R}^{3}$ be the set parameter points $(a, b, c)$ such that

$$
\begin{aligned}
& a^{2}+b^{2}>c, \\
& x_{i}^{2}+y_{i}^{2}-2 a x_{i}-2 b y_{i}+c \neq 0, \quad\left(x_{i}, y_{i}\right) \in S .
\end{aligned}
$$

The closure of a path-connected subset of $U$ is called a domain of separating circles.

The set $U$ is an open set consisting of multiple disconnected subsets, which may be bounded or unbounded. If $C$ is any circle not passing through the points of $S$, then the unique domain in which its parameters lie will be denoted as $D(C)$.

It is not difficult to see that the path-connected subsets of $U$ are exactly the families of circles that always separate the set $S$ in the same two parts. According to Definition 2 the subset $U$ consists of all the points in $\mathbb{R}^{3}$ for which

$$
x_{i}^{2}+y_{i}^{2}-2 a x_{i}-2 b y_{i}+c \neq 0
$$

for $\left(x_{i}, y_{i}\right) \in S$. If we let $(a, b, c)$ vary along a continuous path $(a(t), b(t), c(t)), t \in$ $[0,1]$ then if a point $p$ lies inside the circle defined by $(a(0), b(0), c(0))$ it will also be inside the circle defined by $(a(1), b(1), c(1))$ since a path cannot contain circles that pass through $p$. Similarly, exterior points will remain exterior along the path. Therefore, the connected sets in $U$ have the form

$$
\begin{aligned}
& a^{2}+b^{2}>c, \\
& x_{i}^{2}+y_{i}^{2}-2 a x_{i}-2 b y_{i}+c>0, \quad\left(x_{i}, y_{i}\right) \in \sigma_{S}^{-1}(+), \\
& x_{j}^{2}+y_{i}^{2}-2 a x_{i}-2 b y_{i}+c<0, \quad\left(x_{i}, y_{i}\right) \in \sigma_{S}^{-1}(-),
\end{aligned}
$$

where $\sigma_{S}$ is a circular separation of $S$. Since a domain was defined as the closure of a connected subset of $U$, a domain is a set that satisfies the following inequalities:

$$
\begin{aligned}
& a^{2}+b^{2} \geq c, \\
& x_{i}^{2}+y_{i}^{2}-2 a x_{i}-2 b y_{i}+c \geq 0, \quad\left(x_{i}, y_{i}\right) \in \sigma_{S}^{-1}(+), \\
& x_{j}^{2}+y_{i}^{2}-2 a x_{i}-2 b y_{i}+c \leq 0, \quad\left(x_{i}, y_{i}\right) \in \sigma_{S}^{-1}(-) .
\end{aligned}
$$

We shall denote this domain as $D\left(\sigma_{S}\right)$.

\subsection{Polyhedral and Polytopal Domains}

It is often assumed that a domain of separating circles is always polyhedral. For a general set $S$ this is not always true, however. We shall make this more precise. The equation $a^{2}+b^{2}=c$ defines a paraboloid [7, 8]. For each point $\left(x_{i}, y_{i}\right)$ in $S$, the equation

$$
x_{i}^{2}+y_{i}^{2}-2 a x_{i}-2 b y_{i}+c=0
$$

represents a plane tangent to this paraboloid. The tangent point is $\left(a, b, a^{2}+b^{2}\right)$. Halfspaces of the form $x_{i}^{2}+y_{i}^{2}-2 a x_{i}-2 b y_{i}+c \geq 0$ correspond to points of $S^{+}$, and are always oriented towards the paraboloid. Halfspaces that correspond to points of $S^{-}$are oriented away from the paraboloid.

There is also an unbounded non-polyhedral domain which consists of the circles that do not contain any of the points of $S$, that is, $S^{+}=S$ and $S^{-}=\emptyset$. This domain is not polyhedral as one of its boundary surfaces is the paraboloid $c=a^{2}+b^{2}$. All other domains are polyhedral, however. 
Proposition 1. Let $S$ be a finite set. Let $C$ be a circle not passing through any point of $S$, giving rise to a circular separation $\sigma_{S}$. Then $D(C)$ is a $H$-polyhedron if and only if $S^{-}$is non-empty. Furthermore, $D(C)$ is a polytope if conv $S^{+} \cap \operatorname{conv} S^{-}$contains a non-empty open set.

Proof. First, if $S^{-}$contains a single point $p_{1}$, then the intersection of $D(C)$ with the surface $c=a^{2}+b^{2}$ contains exactly one point, which represents the circle with radius $r=0$ centered at $p_{1}$. If $S^{-}$contains more than one point, each circle must have a strictly positive radius, and the intersection of $D(C)$ with the paraboloid $c=a^{2}+b^{2}$ is therefore empty. In either case, we can discard the inequality $a^{2}+b^{2}>c$. Hence, $D(C)$ is a $H$-polyhedron, that is, the intersection of a finite set of closed half-spaces.

Second, the set defined by (4) is bounded provided there are no parameter points for circles with infinite radius. For this it suffices that $S^{+}$cannot be separated from $S^{-}$by a straight line, even if we discard the points on that line. This is equivalent to stating that $\operatorname{conv} S^{+} \cap \operatorname{conv} S^{-}$contains a non-empty open set.

Both conditions of Proposition 1 are satisfied if the interior of the convex hull of $S^{+}$ contains at least one of the points of $S^{-}$. Therefore, the simplest example of a polytopal domain is when $S^{+}$contains three points $p_{1}, p_{2}, p_{3}$, and $S^{-}$a fourth point $p_{4}$ which lies inside the triangle $p_{1} p_{2} p_{3}$. The polytope is then bounded by four planes. These planes delimit four halfspaces, three oriented towards the paraboloid, and one oriented away from the paraboloid.

\subsection{Polytopal Domains and Elementary Circular Separations}

There is a direct relation between elementary circular separations and domains. Since an elementary circular separation can be extended unambigously to a global separation, we define $D\left(\sigma_{R}\right)$ as $D\left(\sigma_{R}\right)=D\left(\sigma_{S}\right)$ where $\sigma_{S}$ is the extension of $\sigma_{R}$. Assume furthermore, that the domain $D\left(\sigma_{R}\right)$ is polytopal. Then each face of the polytope corresponds to a point of $S$, i.e., a face lies in a plane of the form

$$
x_{i}^{2}+y_{i}^{2}-2 a x_{i}-2 b y_{i}+c=0
$$

where $\left(x_{i}, y_{i}\right) \in S$. Similarly, each vertex of the polytope corresponds to an elementary circular separation. To be precise, a vertex lies at the intersection of three or more parameter planes of the form

$$
\begin{aligned}
& x_{1}^{2}+y_{1}^{2}-2 a x_{1}-2 b y_{1}+c=0, \\
& \cdots \\
& x_{n}^{2}+y_{n}^{2}-2 a x_{n}-2 b y_{n}+c=0,
\end{aligned}
$$

where $\left(x_{1}, y_{1}\right), \ldots,\left(x_{n}, y_{n}\right)$ lie on a common circle. By attributing signs to the points we can establish an elementary circular separation consistent with Definition 11. The relative position of the vertex with respect to the polytope determines the sign of each point. If the polytope is contained in the halfspace $x_{1}^{2}+y_{1}^{2}-2 a x_{1}-2 b y_{1}+c \geq 0$, then $\left(x_{1}, y_{1}\right)$ receives a positive sign, otherwise $\left(x_{1}, y_{1}\right)$ receives a negative sign.

An edge of the polytope always corresponds to a family of circles that have two points in common, as we can verify by explicit calculation. To be precise, the edge 
that connects a vertex $\left(a_{1}, b_{1}, c_{1}\right)$ with a vertex $\left(a_{2}, b_{2}, c_{2}\right)$, corresponds to a family of circles of the form

$$
x^{2}+y^{2}-2\left(a_{1}(1-t)+a_{2} t\right) x-2\left(b_{1}(1-t)+b_{2} t\right) y+c_{1}(1-t)+c_{2} t
$$

where $0 \leq t \leq 1$. Since all coincidence relations are preserved by an affine transformation, without loss of generality, we may assume that the vertices are $\left(a_{1}, b_{1}, c_{1}\right)=$ $\left(-1,0, c_{1}\right)$ and $\left(a_{2}, b_{2}, c_{2}\right)=\left(1,0, c_{2}\right)$. The above expression then simplifies to

$$
c_{1}(1-t)+c_{2} t-2(-1+2 t) x+x^{2}+y^{2} .
$$

By letting $t$ take two distinct values $t_{1}$ and $t_{2}$, we find that the intersection points are $\left(1 / 4\left(-c_{1}+c_{2}\right), \pm 1 / 4 \sqrt{-(c 1-c 2)^{2}-8(c 1+c 2)}\right)$. Because these coordinates are independent of $t_{1}$ and $t_{2}$, each circle in (6) passes through the same two points.

Fig. 1 shows an example. The elementary circular separation with $R^{-}=\{(1,1)$, $(2,1)\}$, and $R^{+}=\{(1,0),(2,0)\}$, induces the signs shown in Fig. 1 (a). The gray points belong to $S^{-}$and lie inside the separating circles, the black points belong to $S^{+}$. There are infinitely many circles that separate $S$ into $S^{+}$and $S^{-}$. Fig. 11(a) shows one of these circles. Fig. 1 (b) shows the polytopal domain, which has 5 vertices. The gray area in Fig.1 (c) shows the possible positions of the circle centers, which can be found by projecting the domain onto the $a b$-plane. Each vertex of the domain corresponds to one circle, also shown in Fig. 1(c). Each circle, and therefore each vertex, determines an elementary circular separation.

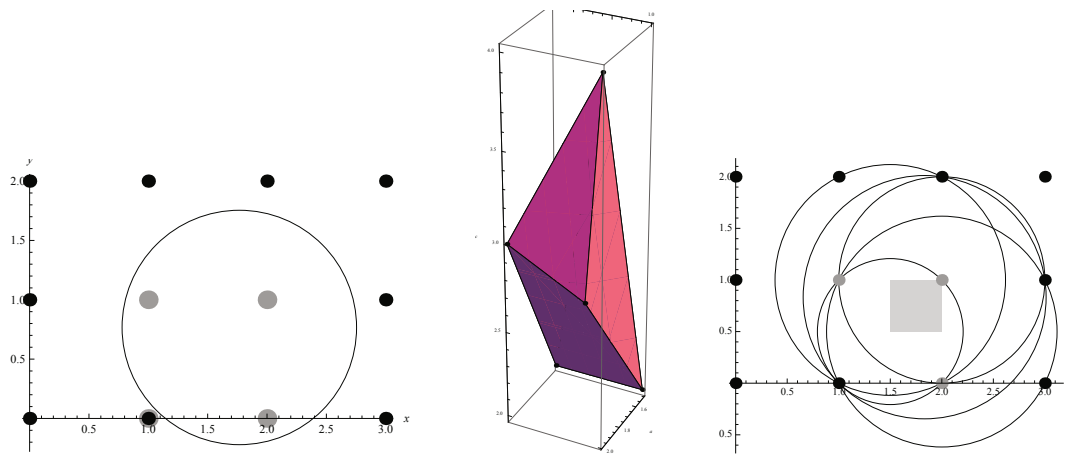

Fig. 1. Family of separating circles

Fig. 2 shows a family of circles whose parameters lie on a polytope edge, all passing through two common points. Clearly, if a planar point $p$ lies inside one of these circles, then $p$ lies in at least one of the two circles that correspond to the vertices of the edge. In other words, the area covered by all the members of the family is the same as the area covered by the two circles of the vertices. This property can be generalized. Let $C_{(a, b, c)}$ denote the circle with parameters $(a, b, c)$.

Proposition 2. Let $\sigma_{S}$ be a circular separation with polytopal domain $D\left(\sigma_{S}\right)$. If a planar point $p$ lies inside one of the circles with parameters in $D\left(\sigma_{S}\right)$, then there is a vertex $v=(a, b, c)$ of $D\left(\sigma_{S}\right)$ such that $p$ lies inside $C_{(a, b, c)}$. 




Fig. 2. Circles that correspond to a polytope edge. All the circles pass through two common points.

Proof. The polytope $D\left(\sigma_{S}\right)$ contains all the circles that separate $S$ into two sets $S^{-}$ and $S^{+}$. The half-space $H$ of circles containing the point $p=\left(x_{0}, y_{0}\right)$ is determined by

$$
x_{0}^{2}+y_{0}^{2}-2 a x_{0}-2 b y_{0}+c<0 .
$$

The point $p$ lies inside one of the circles of $D\left(\sigma_{S}\right)$ provided $H \cap D\left(\sigma_{S}\right) \neq \emptyset$. If $H \cap$ $D\left(\sigma_{S}\right)$ is non-empty, it forms a polytope which contains all the circles that contain $p$. Since $D\left(\sigma_{S}\right)$ is a polytope, $H \cap D\left(\sigma_{S}\right)$ contains at least one vertex $v$ of $D\left(\sigma_{S}\right)$. Hence, the circle $C_{(a, b, c)}$ contains $p$.

Thus the area covered by the interior of the circles in $D\left(\sigma_{S}\right)$ is the same as the area covered by the interior of the circles corresponding to the vertices of $D\left(\sigma_{S}\right)$.

\section{Properties of Elementary Circular Separations}

We prove two additional properties of elementary circular separations. First, determining whether a circular set and a sign map form an elementary circular separation is straightforward.

Proposition 3. Let $\sigma_{R}: R \rightarrow\{+,-\}$ be a map that attributes signs to the points of a circular set $R$. Then the map $\sigma_{R}$ is an elementary circular separation if and only if $\sigma_{R}^{-1}(+)$ can be separated by a straight line from $\sigma_{R}^{-1}(-)$.

Proof. We use the shorthands $R^{+}=\sigma_{R}^{-1}(+)$, and $R^{-}=\sigma_{R}^{-1}(-)$. First we show that the preimages $R^{+}, R^{-}$of an elementary circular separation $\sigma_{R}$ can always be separated by a straight line. Let $D$ be the common circle on which the points lie. Let $L$ be any straight line separating $R^{+}$from $R^{-}$. Then $L$ divides the open disk bounded by $D$ into two parts, $L^{+}$and $L^{-}$, with $R^{+} \subset L^{+}$and $R^{-} \subset L^{-}$. Furthermore, $L$ crosses $D$ in two distinct points $v$ and $w$. Let $q$ denote any point that lies on the bisector of $v$ and $w$ and in $L^{+}$. Then the circle $C$ passing through $v, q, w$ satisfies the conditions required by Definition 1 that is, all the points in $R^{+}$lie outside $C$ and all the points in $R^{-}$lie inside $C$.

Conversely, suppose the points of $R^{+} \cup R^{-}$lie on a common circle, but that $R^{+}$ cannot be linearly separated from $R^{-}$. Since they cannot be linearly separated, the 
intersection of their convex hulls, i.e., $\operatorname{conv} R^{+} \cap \operatorname{conv} R^{-}$, is non-empty. However, since the points lie on a circle, none of the points in $R^{+}$lies in $\operatorname{conv} R^{-}$, and vice-versa. It follows that there are two points $p_{1}, p_{2}$ in $R^{+}$and two points $p_{3}, p_{4}$ in $R^{-}$such that the line segment $p_{1} p_{2}$ crosses the line segment $p_{3} p_{4}$. If there exists a circle $C$ that separates $R^{+}$from $R^{-}$, then $C$ would also separate $p_{1}, p_{2}$ from $p_{3}, p_{4}$. Therefore it is sufficient to prove that even these 4 points cannot be separated by a circle, with $p_{1}, p_{2}$ outside the circle and $p_{3}, p_{4}$ inside the circle.

This part of the proof is illustrated in Fig. 3, which shows four points on a common circle $D$, and the bisectors of the pairs $\left\{p_{1}, p_{3}\right\},\left\{p_{3}, p_{2}\right\},\left\{p_{2}, p_{4}\right\}$, and $\left\{p_{4}, p_{1}\right\}$. These bisectors pass through the circle center and they divide the plane into 4 open disjoint segments $S_{1}, \ldots, S_{4}$. The bisector of $\left\{p_{1}, p_{3}\right\}$ divides the plane into two open halfplanes. If a circle $C$ has to satisfy Definition 1 then $p_{1}$ must lie outside $C$ and $p_{3}$ inside $C$, and the center of $C$ must lie in the half-plane which contains $p_{3}$. Furthermore, since $p_{2}$ must also lie outside $C$, it follows that the center of $C$ must lie in the sector $S_{3}$. On the other hand, by considering $p_{1}, p_{2}$, and $p_{4}$ we find that the center must also lie in the sector $S_{4}$. Since $S_{3} \cap S_{4}=\emptyset$, this is impossible.

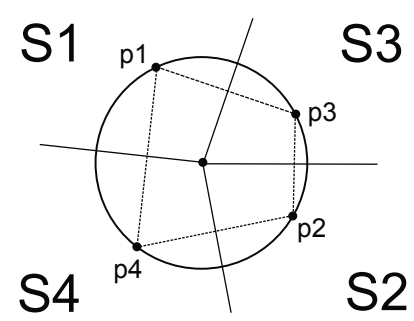

Fig. 3. Illustration of the proof of Proposition 3

With Proposition 3 we can list all elementary circular separation functions that can be defined on a finite circular set. If we divide the points of a circular set into linearly separable parts, these parts always belong to two disjoint circle segments. Hence, each elementary circular separation corresponds to a partitioning of a circular set into two contiguous parts.

O'Rourke et al showed that any largest separating circle of finite radius must either pass through 3 points of $S^{+}$, or it must pass through 2 points of $S^{+}$and one of $S^{-}[7]$. As a related result, we will show that in a domain there is at least one circle passing through 2 points of $S^{+}$and one point of $S^{-}$.

Proposition 4. Let $\sigma_{S}$ be a circular separation with polytopal domain $D\left(\sigma_{S}\right)$. Then $D\left(\sigma_{S}\right)$ always contains a vertex defined by an elementary circular separation $\sigma_{R}$ with $\left|\sigma_{R}^{-1}(+)\right| \geq 2$ and $\left|\sigma_{R}^{-1}(-)\right| \geq 1$.

Proof. We use the shorthands $S^{+}=\sigma_{S}^{-1}(+)$ and $S^{-}=\sigma_{S}^{-1}(-)$. Let $C$ be any circle in the domain $D\left(\sigma_{S}\right)$. We will deform $C$ continuously until its parameters coincide with an elementary circular separation that satisfies the above requirements. We will start by deforming $C$ until it touches two points $p_{1}^{+}, p_{2}^{+}$of $S^{+}$. Then we will deform it further until it touches a third point $p_{3}^{-}$that lies in $S^{-}$. 
Since the domain is bounded, $C$ separates $S$ into two subsets $S^{+}$and $S^{-}$that cannot be linearly separated. Clearly, this is only possible if $\left|S^{-}\right| \geq 1$, and $\left|S^{+}\right| \geq 3$. First, we increase the radius of $C$ until the circle passes through a point $p_{1}$ of $S^{+}$, as shown in Fig. 4(a). This is always possible since $\left|S^{+}\right| \geq 3$.

Second, consider the tangent to $C$ passing through the point $p_{1}$. Since $S^{+}$and $S^{-}$ cannot be separated linearly, there must be at least one other point of $S^{+}$that lies at same side of the tangent as all the points of $S^{-}$. Hence, by moving the center $a$ of $C$ along the line $p_{1} a$ away from $p_{1}$, we can further increase the radius of the circle until it passes through $p_{1}$ and a second point $p_{2}$ of $S^{+}$. Fig. 4 (b) illustrates this.

Finally, we arbitrarily select a point $q$ of $\left|S^{-}\right|$that does not lie on the line $p_{1} p_{2}$. We distinguish two cases.

Case A. The point $q$ lies at the same side from the line $p_{1} p_{2}$ as the center of $C$. Then we start to move the center along the bisector of $p_{1}$ and $p_{2}$ in the direction of $p_{1} p_{2}$ until the circle passes through $p_{1}, p_{2}$ and and $q$ or one of the other points of $S^{-}$. This is illustrated in Fig. 4(c). Note that it may be necessary to move the center to the other side of $p_{1}, p_{2}$.

Case B. The chosen point $q$ lies at the side from the line $p_{1} p_{2}$ which is the opposite of the side of the center of $C$, as shown in Fig. 4(d). In this case we increase the radius of the circle by moving its center $a$ along the bisector of $p_{1}$ and $p_{2}$ away from the line $p_{1} p_{2}$ until $C$ passes through $p_{1}, p_{2}$ and $q$ or one of the other points of $S^{-}$.

In both cases, however, the motion of the center of the circle may stop when the circle touches a point of $S^{+}$before it reaches a point of $S^{-}$. First, consider how this can happen in case A. Fig. 4(e) shows that the circle has hit a third point $p_{3}$ of $S^{+}$before it reaches $q$. Note that this point must always lie at the side of $p_{1} p_{2}$ that is opposite to $q$. If this happens, it suffices to replace the chord $p_{1} p_{2}$, by one of the smaller chords, for example $p_{1} p_{3}$, and continue the shrinking process along the bisector of the new chord. If we then hit another point of $S^{+}$we can keep replacing the chords by smaller chords. Since $S^{+}$is finite this process must end until we hit one of the points in $S^{-}$. Second, we consider how this can happen in case B. Fig. 4(f) shows that the circle has hit a point $p_{3}$, which must lie at side of $p_{1} p_{2}$ that is opposite to $q$. Clearly, $q$ lies at the same side of the line $p_{2} p_{3}$ as the center of the circle. That is, we can replace the chord $p_{1} p_{2}$ by the chord $p_{2} p_{3}$ and continue as in case $\mathrm{A}$.

In either case, we can deform the circle until it passes through two points $p_{1}$ and $p_{2}$ of $S^{+}$and one point of $S^{-}$.

In the example shown in Fig. 1, three of the 5 vertices satisfy the conditions of Proposition 4. There is also a vertex with $\left|R^{+}\right|=3,\left|R^{-}\right|=0$. One of these four vertices yields the largest separating circle.

\section{Distances and Geometric Properties}

Once the domains of the families of separating circles have been calculated several geometric properties can be verified immediately, since they directly relate to the topological relations between the polytopal domains. For example, given two families of circles there exists a pair of concentric circles, one from each family, provided the circle centers of the two families intersect. This section considers a more involved computation: 


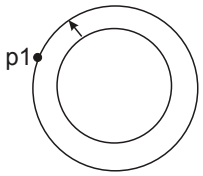

(a)

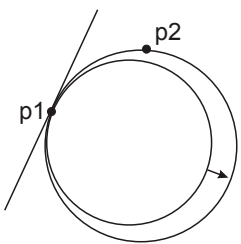

(b)

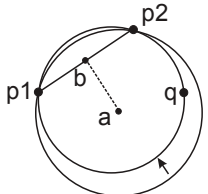

(c)

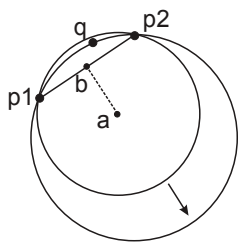

(d)

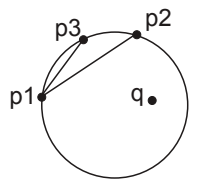

(e)

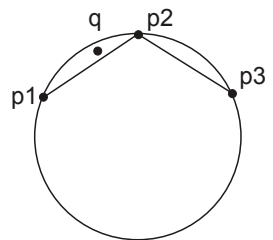

(f)

Fig. 4. Illustration of the proof of Proposition 4

the distance between a family of circles and a point. The distance between a point $p$ and a circle $C$ is defined as the shortest distance between $p$ and any point $q$ on $C$, and will be denoted as $d(p, C)$. If $D\left(\sigma_{S}\right)$ is a polytopal domain of circles, then we define the maximum distance between a point $p$ as $\max _{(a, b, c) \in D\left(\sigma_{S}\right)} d\left(p, C_{(a, b, c)}\right)$. That is, as maximum of the distances between $p$ and any circle in $D\left(\sigma_{S}\right)$. Likewise, the minimum distance is defined as $\min _{(a, b, c) \in D\left(\sigma_{S}\right)} d\left(p, C_{(a, b, c)}\right)$. We will prove that the maximum distance can be found by examining only the circles that correspond to the vertices of a domain. We start with a simplified case.

Lemma 1. Let $V=\left\{\left(a_{1}, b_{1}, c_{1}\right),\left(a_{2}, b_{2}, c_{2}\right)\right\}$ be a set of two parameter points, and let $P$ denote their convex span. Let $p$ be any point in the plane. Then there is a point $u \in C_{\left(a_{1}, b_{1}, c_{1}\right)} \cup C_{\left(a_{2}, b_{2}, c_{2}\right)}$ such that

$$
\max _{(a, b, c) \in P} d\left(C_{(a, b, c)}, p\right)=d(u, p) .
$$

Furthermore, if $p$ does not lie on one of the circles of $P$, then there is a point $w \in$ $C_{\left(a_{1}, b_{1}, c_{1}\right)} \cup C_{\left(a_{2}, b_{2}, c_{2}\right)}$ such that $\min _{(a, b, c) \in P} d\left(C_{(a, b, c)}, p\right)=d(w, p)$.

Proof. The proof follows from simple geometric considerations, illustrated in Fig. 5 Fig. 5. a) shows two circles, one centered around $c_{1}$ and one around $c_{2}$, and an ellipse passing through $q_{1}$ and $q_{2}$. This ellipse is part of the locus of points that are equidistant to both circles. This locus also comprises a hyperbola (not shown here). The ellipse consists of those equidistant points that lie inside one circle but outside the other circle.

The family of circles with parameters in $P$ consists of circles that pass through the points $q_{1}$ and $q_{2}$, and can be represented as

$$
x^{2}+y^{2}-2\left(a_{1}(1-t)+a_{2} t\right) x-2\left(b_{1}(1-t)+b_{2} t\right) y+c_{1}(1-t)+c_{2} t
$$

with $0 \leq t \leq 1$. With $q_{1}, q_{2}, c_{1}, c_{2}$ we construct 5 straight line segments. Together with the ellipse they divide the plane into 8 distinct regions, shown in Fig.5(b). For example, region $R_{7}$ is bounded by the ellipse, and the lines $c_{2} q_{1}$ and $c_{2} q_{2}$. 
The maximum distance between a planar point $p$ and the family of circles (7) depends on the region in which $p$ lies. When $p$ lies either in $R_{3}$ or $R_{4}$ the maximum distance between $p$ and (7) is the distance between $p$ and $q_{1}$. For example, when $p$ lies in $R_{4}$, this maximum is attained by a circle whose center lies on the straight line passing through $p$ and $q_{1}$. In that case, the circle lies "behind" the point $q_{1}$ as seen from $p$. Similarly, when $p$ lies either in $R_{1}$ or $R_{2}$, the maximum distance is equal to distance between $p$ and $q_{2}$. When $p$ lies in either $R_{6}$ or $R_{8}$, the maximum distance between $p$ and (7) is the same as the distance between $p$ and the circle centered around $c_{1}$. In fact, this is the furthest a circle in this family can move away from $p$. Finally, when $p$ lies in either $R_{5}$ or $R_{7}$, the maximum distance is equal to the distance between $p$ and the circle centered around $c_{2}$.

In all the cases considered the maximum distance is given by either the distance to the circle in (7) for which $t=0$, that is $C_{\left(a_{1}, b_{1}, c_{1}\right)}$, or for which $t=1$, that is $C_{\left(a_{2}, b_{2}, c_{2}\right)}$, or by the distance to one of the two points $q_{1}$ or $q_{2}$ which are common to both circles.

For the minimum distance, the proof proceeds in a similar way. We only give a brief sketch. Fig. 5 (c) illustrates the proof when the polytope consists of a line segment between the two parameter points $\left(a_{1}, b_{1}, c_{1}\right)$ and $\left(a_{2}, b_{2}, c_{2}\right)$. Fig. 5 (c) shows how the two circles, and their equidistant ellipse and equidistant hyperbola divide the plane in 8 regions. When $p$ either lies in $R_{2}, R_{3}, R_{6}$ or $R_{7}$ there is a circle of the form (7) passing through $p$. This case is excluded from the theorem. If $p$ does not lie on a circle of $P$, then $p$ either lies in $R_{1}, R_{4}, R_{5}$ or $R_{8}$. When $p$ lies in $R_{1}$ or $R_{5}$, the closest circle is $C_{\left(a_{1}, b_{1}, c_{1}\right)}$. If $p$ lies in $R_{4}$ or $R_{8}$, the closest circle is $C_{\left(a_{2}, b_{2}, c_{2}\right)}$. Hence, the minimum distance is either zero, or equal to $\min \left(d\left(p, C_{\left(a_{1}, b_{1}, c_{1}\right)}\right), d\left(p, C_{\left(a_{2}, b_{2}, c_{2}\right)}\right)\right)$.

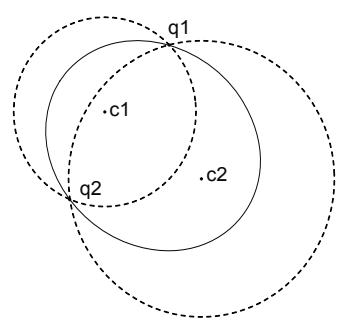

(a)

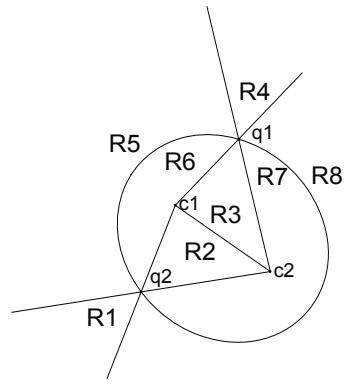

(b)

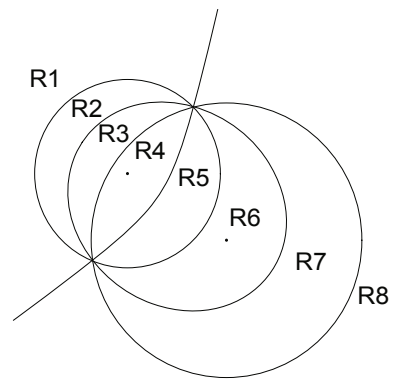

(c)

Fig. 5. (a) Two circles shown with dashed lines, and an ellipse of points that are equidistant to both circles. (b) The ellipse and straight lines through the centers and the common points divide the plane into 8 regions.

We now extend this result to a more general setting.

Theorem 1. Let $\sigma_{S}$ be a circular separation with polytopal domain $D\left(\sigma_{S}\right)$. Let $V$ be set of vertices of $D\left(\sigma_{S}\right)$, and let $W$ denote the set $\bigcup_{(a, b, c) \in V} C_{a, b, c}$. Let $p$ be any point in the plane. Then there is a point $u$ in $W$ such that $\max _{(a, b, c) \in D\left(\sigma_{S}\right)} d\left(C_{(a, b, c)}, p\right)=$ $d(u, p)$. Furthermore, if $p$ does not lie on one of the circles of $D\left(\sigma_{S}\right)$, then there is a point $w$ in $W$ such that $\min _{(a, b, c) \in D\left(\sigma_{S}\right)} d\left(C_{(a, b, c)}, p\right)=d(w, p)$. 
Proof. We use the shorthand $S^{+}=\sigma_{S}^{-1}(+)$ and $S^{-}=\sigma_{S}^{-1}(-)$. It suffices to show that the point $u$ always lies on one of the circles $C_{(a, b, c)}$ where $(a, b, c) \in V$. Let $\left(a_{0}, b_{0}, c_{0}\right) \in D\left(\sigma_{S}\right)$ be the parameters of a circle for which we have $d\left(C_{\left(a_{0}, b_{0}, c_{0}\right)}, p\right)$ $=\max _{(a, b, c) \in P} d\left(C_{(a, b, c)}, p\right)$. We will show that we can always replace $C_{\left(a_{0}, b_{0}, c_{0}\right)}$ by a circle $C_{(a, b, c)}$, with $(a, b, c) \in V$ whose distance to $p$ is at least as large as that of $C_{\left(a_{0}, b_{0}, c_{0}\right)}$. Showing that $(a, b, c) \in V$ is equivalent to showing that $C_{(a, b, c)}$ contains at least three points of $S^{+} \cup S^{-}$. We start by assuming that $C_{\left(a_{0}, b_{0}, c_{0}\right)}$ does not contain any point of $S^{+} \cup S^{-}$. Then we can always move the center $\left(a_{0}, b_{0}\right)$ away from $p$ along the line passing through $\left(a_{0}, b_{0}\right)$ and $p$. If the radius is kept constant the distance will increase. Since this is impossible, $C_{\left(a_{0}, b_{0}, c_{0}\right)}$ contains at least one point of $S^{+} \cup S^{-}$.

Next, we assume that $C_{\left(a_{0}, b_{0}, c_{0}\right)}$ contains just one point $q_{1}$ of $S^{+} \cup S^{-}$. Let $L$ denote the line passing through $\left(a_{0}, b_{0}\right)$ and $p$. First, suppose $q_{1}$ lies on $L$. Then it is clear that we can move the center along $L$ while keeping the distance to $p$ fixed until we hit a second point of of $S^{+} \cup S^{-}$. Second, suppose $q_{1}$ does not lie on $L$. Let $q{ }_{1}$ denote the mirror image of $q_{1}$ by the reflection across $L$. We define a pencil of circles of the form (7) with common points $q_{1}, q^{\prime}{ }_{1}$ and center on $L$. Let $q_{3}$ be the first point of $S^{+} \cup S^{-}$ that we hit when we move in this pencil $\left(a_{0}, b_{0}\right)$ away from $p$, and let $q_{4}$ be the first point of $S^{+} \cup S^{-}$hit when we move $\left(a_{0}, b_{0}\right)$ towards from $p$. Since $P$ is bounded both points must exist. Let $C_{\left(a_{1}, b_{1}, c_{1}\right)}$ be the circle passing through $q_{1}, q^{\prime}{ }_{1}, q_{3}$ and let $C_{\left(a_{2}, b_{2}, c_{2}\right)}$ denote the circle passing through $q_{1}, q^{\prime}{ }_{1}, q_{4}$. According to Lemma 1 the maximal distance between $p$ and the pencil of circles is either the distance to $C_{\left(a_{1}, b_{1}, c_{1}\right)}$, to $C_{\left(a_{2}, b_{2}, c_{2}\right)}$ or to one of the points $q_{1}, q^{\prime}{ }_{1}$. However, $q_{1}, q^{\prime}{ }_{1}$ lie at the same distance of $p$, and $q_{1}$ is a point of $S^{+} \cup S^{-}$. Hence, we can replace $C_{\left(a_{0}, b_{0}, c_{0}\right)}$ always by a circle that contains two points $q_{1}, q_{2}$ of $S^{+} \cup S^{-}$, and state that the maximal distance is either the distance to this circle or one of the points of $S^{+} \cup S^{-}$.

Finally, we can repeat the previous argument, but now for the pencil of circles defined by the common points $q_{1}, q_{2}$, until we hit a third point of $S^{+} \cup S^{-}$. The maximal distance is either the distance to this new circle or to one of the points $q_{1}, q_{2}$, which are both in $S^{+} \cup S^{-}$. For minimum distances, the proof is similar.

The above result also shows how an algorithm can compute the correct maximum or minimum distance. First, for each edge of the polytope we compute the maximum distance between $p$ and the circles of the edge. This can be done by examining the position of $p$ with respect to the straight lines shown in Fig. 5, where the circles correspond to the vertices adjacent to the edge. If $p$ lies in one of the regions $R_{1}, \ldots, R_{4}$ the maximum distance is either $d\left(p, q_{1}\right)$ or $d\left(p, q_{2}\right)$. Otherwise, it is the distance to one of the two circles corresponding to the vertices. After this distance has been computed for all the edges, it suffices to take the global maximum.

To find the minimum distance one first has to verify whether $p=\left(x_{p}, y_{p}\right)$ does not lie on one the circles in $P$. It suffices to verify whether the plane

$$
x_{p}^{2}+y_{p}^{2}-2 a x_{p}-2 b y_{p}+c=0
$$

crosses the polytope $P$. If $p$ does not lie on any of the circles, the distance can be found by computing the distance for each circle corresponding to a vertex, and by taking the global minimum. 


\section{Time Complexity and Conclusion}

Clearly, the computation of the distance between a point and the separating circles depends mainly on the computation of the complete face lattice (faces, edges, and vertices) of the parameter polytope. Clarkson and Shor give a randomized algorithm in 3D with expected time complexity of $O(n \log n)$, where $n$ is the number of half-spaces. This algorithm was derandomized by Chazelle [1, 2]. The polytope of separating circles for $|S|=n$ can therefore be computed in $O(n \log n)$ time, where $O(n \log n)$ is an upper bound for the expected number of edges, as well as vertices in the face lattice. The computation of the maximal and minimal distances requires the examination of all edges and vertices, where each vertex or edge can be processed in constant time. Hence distances can be computed in $O(n \log n)$ time.

\section{References}

1. Chazelle, B.: An optimal convex hull algorithm in any fixed dimension. Discrete and Computational Geometry 10, 377-409 (1993)

2. Clarkson, K.L., Shor, P.: Applications of random sampling in computational geometry, II. Discrete Computational Geometry 4, 387-421 (1989)

3. Coeurjolly, D., Gerard, Y., Reveilles, J.-P., Tougne, L.: An elementary algorithm for digital arc segmentation. Discrete Applied Mathematics 139, 31-50 (2004)

4. Damaschke, P.: The linear time recognition of digital arcs. Pattern Recognition Letters 16, 543-548 (1995)

5. Fisk, S.: Separating points sets by circles, and the recognition of digital disks. IEEE Transactions on Pattern Analysis and Machine Intelligence 8, 554-556 (1986)

6. Kim, C.E., Anderson, T.A.: Digital disks and a digital compactness measure. In: Annual ACM Symposium on Theory of Computing, pp. 117-124 (1984)

7. O'Rourke, J., Koraraju, S.R., Meggido, N.: Computing circular separability. Discrete and Combinatorial Geometry 1, 105-113 (1986)

8. Roussillon, T., Tougne, L., Sivignon, I.: On three constrained versions of the digital circular arc recognition problem. In: Brlek, S., Reutenauer, C., Provençal, X. (eds.) DGCI 2009. LNCS, vol. 5810, pp. 34-45. Springer, Heidelberg (2009) 